
Revue
de l'histoire des religions
Revue de l'histoire des religions
2 | 2007
Divination et révélation dans les mondes grec et romain

\title{
Plutarque et la divination : la piété d'un prêtre philosophe
}

Plutarch and divination. Piety of a priest and philosopher as well

Dominique Jaillard

\section{(2) OpenEdition \\ Journals}

Édition électronique

URL : http://journals.openedition.org/rhr/5266

DOI : $10.4000 /$ rhr.5266

ISSN : 2105-2573

Éditeur

Armand Colin

Édition imprimée

Date de publication : 1 avril 2007

Pagination : 149-169

ISBN : 978-2200-92333-4

ISSN : 0035-1423

Référence électronique

Dominique Jaillard, «Plutarque et la divination : la piété d'un prêtre philosophe », Revue de l'histoire des religions [En ligne], 2 | 2007, mis en ligne le 01 juin 2010, consulté le 01 mai 2019. URL : http:// journals.openedition.org/rhr/5266 ; DOI : 10.4000/rhr.5266 


\section{Plutarque et la divination : la piété d'un prêtre philosophe}

Les spéculations de Plutarque philosophe sur la divination s'ancrent dans l'expérience et le savoir de ritualiste du prêtre de Delphes, dans les questions que le processus mantique pose au praticien (une pythie est morte par suite du non-respect des règles de consultation). Son examen critique des théories philosophiques et les hypothèses qu'il propose dans un cadre médio-platonicien s'apprécient à l'aune d'une pratique rituelle et d'une piété qui, fondées sur le respect des traditions, suscitent une dynamique spéculative souvent novatrice, par exemple dans l'esquisse d'une théorie générale des signes et des manifestations divines. En réfléchissant les enjeux du rite, en en produisant l'exégèse, le philosophe accomplit la piété ancestrale.

\section{Plutarch and divination. Piety of a priest and philosopher as well}

Plutarch speculated on divination on the basis of his own Delphic, priestly experience. His account of a Pythia's death through disrespect of the rules of oracular consultation is that of a man involved in ritual knowledge and duties. His Middle-Platonic critical examination of philosophical theories partly relies on rite and piety as constituents of tradition. Ritual concern and philosophical training are thus closely linked in producing a dynamical understanding of divination for which Plutarch's attempt at sketching a general theory of signs and divine manifestations provides an example. Pursuing of philosophical Truth may be considered as the highest form of hosiotes because of the light it shades on ritual enactment. In the new garb of the philosopher-priest, Plutarch was acting for the accomplishment of ancestral piety. 
Pendant une trentaine d'années au moins, Plutarque fut un des deux prêtres d'Apollon à Delphes ${ }^{1}$. On en tire rarement toutes les conséquences lorsqu' on considère ses écrits sur la divination ${ }^{2}$ ou les innombrables histoires d'oracle qui émaillent son œuvre. Plutarque est plus volontiers convoqué en témoin de la renaissance de Delphes au tournant des $\mathrm{I}^{\mathrm{er}}$ et $\mathrm{II}^{\mathrm{e}} \mathrm{s}$. ap. J.-C. qu'en praticien des rites mantiques. Archéologues et historiens ont reconnu la valeur documentaire du corpus, quitte à regretter que les informations soient plus aptes à éclairer la Delphes du Haut Empire qu'à clarifier les périodes plus anciennes de l'histoire du sanctuaire. Une autre approche envisage la réflexion de Plutarque sur la divination au plan d'une « sophistique » philosophique vouée à rendre raison des processus oraculaires en discutant les hypothèses des différentes écoles. De ce point de vue, on s'est efforcé, souvent remarquablement, de reconstituer sa pensée «personnelle», philosophique, religieuse ${ }^{3}$ ou politique $^{4}$

1. Pour une introduction à la vie de Plutarque, voir Jean Sirinelli, Plutarque, Paris, Fayard, 2000 (sur le prêtre de Delphes, voir p. 198 et ss.) ; pour le fonctionnement du sanctuaire et son personnel, Georges Roux, Delphes, son oracle et ses dieux, Paris, Les Belles Lettres, 1976 et Stella Georgoudi, «Le porteparole des dieux : Réflexions sur le personnel des oracles grecs », Sibille e linguaggi oracolari. Mito Storia Tradizione. Atti del convegno Macerata-Norcia Settembre 1994, Ileana Chirasi Colombo et Tullio Seppili (éds), Pise-Rome, Istituti editoriali e poligrafici internazionali, 1998, p. 315-365.

2. En premier lieu, les Dialogues pythiques, Sur la disparition des oracles et Pourquoi la Pythie ne rend plus ses oracles en vers, que nous citons et abrégeons selon l'usage, respectivement de defectu et de Pyth. or. Les titres et les fragments de livres perdus (Recueil d'oracles, Sur la descente dans l'antre de Trophonios, Sur la mantique, Sur l'utilité de connaître l'avenir) attestent la place de la divination dans les recherches historiques et dans la réflexion de Plutarque.

3. Quelques jalons : Robert Flacelière, «Plutarque et la Pythie », Revue des Études Grecques, 56, 1943, p. 72-111; Daniel Babut, «La composition des Dialogues pythiques et le problème de leur unité », Journal des savants, 27, 1992, p. 189-234 (repris dans Parerga : choix d'articles 1974-1994, Lyon, Maison de l'Orient méditerranéen, 1994, p. 457-504) ; Giulia Sfameni Gasparro, «Plutarco e la religione delfica : il dio 'filosofo' e il suo esegeta », Plutarco e la Religione, Italo Gallo (éd.), Naples, M. d'Auria, 1995, p. 157-188 ; Jan Opsomer, "Divination and Academic 'Scepticism' according to Plutarch", Plutarchea Lovaniensia. A Miscellany of Essays on Plutarch, Luc van der Stockt (éd.), Louvain, Peeters, 1996, p. 165-194.

4. Philip A. Stadter, "Plutarch and Apollo of Delphi", Gott und die Götter bei Plutarch, Rainer Hirsch-Luipold (éd.), Berlin, Walter de Gruyter, 2005, p. 197-214. 
- une pensée dont on s'accorde aujourd'hui à reconnaître la fermeté et la subtilité ${ }^{5}$ - ou de la situer dans une histoire plus large de la religion et de la philosophie grecques ${ }^{6}$. Mais c'est oublier que le notable qui, comme prêtre, était responsable de l'administration du sanctuaire d'Apollon - le financement lui incombait en partie, la prêtrise étant une liturgie -, et qui a joué un rôle considérable dans la restauration de son prestige - les évergésies d'Hadrien semblent avoir marqué le couronnement de sa carrière delphique ${ }^{7}$ - était aussi un praticien du rituel, un acteur du processus oraculaire.

\section{Activités, savoirs et perplexités du prêtre Plutarque}

Quelques allusions, dont on comprend la relative discrétion dans des dialogues ou des traités de nature essentiellement philosophique, laissent entrevoir l'activité rituelle du prêtre et les questions qui pouvaient concrètement se poser au praticien du processus divinatoire. Dans le petit dialogue si la politique est l'affaire des vieillards,

5. Ainsi que l'atteste, emblématiquement, le titre de Paul Veyne : «Les problèmes religieux d'un païen intelligent : Plutarque », L'empire gréco-romain, Paris, Le Seuil, 2005, p. 633-681.

6. Voir, par exemple Victor Goldschmidt, «Les thèmes du de defectu oraculorum de Plutarque », Revue des Études Grecques, 61, 1948, p. 298 et Questions platoniciennes, Paris, Vrin, 1970, p. 223 : Le de defectu «éclaire tout un chapitre de l'histoire de la religion et de la pensée grecques, et, par ses larges emprunts faits aux traditions antérieures, constitue une source précieuse pour notre connaissance de doctrines et de croyances qui, parfois, remontent au-delà de Platon ».

7. de Pyth or $409 \mathrm{~B}-\mathrm{C}$ : « mais à nous, ce sont des signes plus magnifiques, plus forts et plus clairs que le dieu prodigue, en créant pour ainsi dire, d'un sol auparavant desséché, pauvre et désertique, l'abondance, la magnificence et la gloire (timé). Sans doute, je m'applaudis pour ma part, d'avoir contribué avec zèle au succès de ces entreprises, en compagnie de Polycratès et de Pétraios, et j'applaudis celui qui a été notre guide dans la conduite de ces affaires, qui luimême en médite et en prépare presque tous les détails [l'empereur Hadrien César]. » Sur la restitution du nom d'Hadrien, voir Robert Flacelière, Comptes rendus de l'Académie des Inscriptions et Belles Lettres, 1971, p. 168-185; les lettres impériales confirment cette hypothèse qui nous semble de loin préférable à celle de Saul Levin, "Plutarch's part in the damnatio memoriae of the Emperor Domitian", La Béotie antique, Paris, Éditions du CNRS, 1985, p. 283287, qui suggérait la restitution Domitien. 
Plutarque résume quelques-uns des devoirs qui lui incombaient en tant que prêtre: «Tu sais bien que je sers Apollon depuis maintes Pythiades, mais tu ne viendrais pas me dire : "Plutarque, tu as à ton actif assez de sacrifices, assez de processions, assez de chœurs. Il est temps, maintenant que tu es vieux que tu déposes la couronne et que tu quittes l'oracle pour raison d'âge". ${ }^{8}$ Ailleurs, il précise les fonctions communes aux prêtres et aux Hosiö̈ lors de l'accomplissement du sacrifice préliminaire (prothuómenoi) à la consultation de la Pythie : il évoque l'action rituelle à laquelle il aura présidé chaque fois qu'il officiait dans le sanctuaire, année après année, couronnant les victimes, les aspergeant (kataspéndontes), examinant leurs mouvements et leurs frissons (tèn kínesin autoû kaì tòn trómon apotheoreîn) afin de déchiffrer les signes donnés par le dieu (hemâs semeîa didónai tòn theòn $)^{9}$. Le sacrifice préliminaire a en lui-même une valeur mantique, il indique si Apollon est disposé à répondre, ou du moins si toutes les conditions sont remplies pour que la Pythie puisse officier. Sous la rhétorique de Plutarque pointe un savoir pratique complexe, relatif à la pureté des victimes sacrificielles, aux critères spécifiques à chaque espèce. Le texte présuppose toute une tradition d'exégèse rituelle qui n'est pas exposée pour elle-même, mais mobilisée dans une discussion philosophique où l'opposition, que les modernes - tant philosophes qu'historiens des religions voudraient volontiers drastique, entre le savoir jugé formel du ritualiste et les spéculations du philosophe, tend à s'estomper. « Il convient que la bête de sacrifice soit pure (katharón), saine (asinés) et intacte (adiáphthoron) pour l'âme comme pour le corps. En ce qui concerne le corps, les indices d'un tel état ne sont pas du tout difficiles à discerner ; quant à l'âme, de même qu'on l'examine en présentant aux taureaux de la farine et aux verrats ${ }^{10}$ des pois chiches et que, si

8. $792 \mathrm{~F}$.

9. de defectu $437 \mathrm{~A}$.

10. Káprois, les traductions (Robert Flacelière, Frédérique Ildefonse) donnent habituellement sangliers, ce qui ne fait pas sens dans le contexte sacrificiel en cause, il s'agit de porcs domestiques, et, ainsi que nous le confirme Stella Georgoudi, du verrat, le mâle reproducteur (en parallèle au taureau). Voir, par exemple, Franciszek Sokolowski, Lois Sacrées des Cités Grecques, Paris, 1969, $\mathrm{n}^{\circ}$ 65, 1. 69 ; Supplementum Epigraphicum Graecum, XXXI, 122, 1. 38... 
ces animaux n'y touchent pas, on les considère comme n'étant pas en bonne condition, de même on pense que la chèvre s'éprouve par l'eau froide et que son âme n'est pas dans une disposition conforme à la nature lorsque, sous l'aspersion, elle reste insensible et immobile (apathés kaì akíneton). ${ }^{11}$

Le caractère allusif du propos et la tradition humaniste qui a longtemps dominé les études grecques expliquent que, si les informations factuelles et les spéculations philosophiques de Plutarque ont été prises en compte par la recherche contemporaine, son savoir de ritualiste, son expérience de prêtre, sa réflexion sur sa propre pratique ont été largement sous-évalués dans l'appréciation de sa compréhension des processus mantiques. Même si à Delphes, ce sont les prophètes et les Hosiö̈ qui sont investis du rôle décisif au moment où le dieu s'empare de la Pythie pour énoncer par sa bouche la réponse oraculaire, les prêtres ${ }^{12}$ sont au cœur du dispositif mantique à l'administration duquel ils président. L'enthousiasme ${ }^{13}$ de la Pythie, la lumière divine qui, dans son âme, éclaire l'avenir ${ }^{14}$, sont pour Plutarque de l'ordre du fait, avéré, indiscutable, quoique les modalités de l'inspiration puissent demeurer largement énigmatiques et quelquefois difficiles à maîtriser pour les prêtres et les prophètes en charge du processus rituel. Un accident tragique survenu quelques années au plus avant que Plutarque ne devînt lui-même prêtre, alors que son collègue et ami le prophète Nicandre était déjà en charge, témoigne des problèmes auxquels était confronté le personnel de l'oracle. Une pythie est morte d'une consultation au cours de laquelle on a quelque peu joué avec les règles rituelles en vigueur,

11. de defectu 437 A-B, trad. Robert Flacelière modifiée.

12. S'il ne se confond pas avec le prophète, ce que le témoignage de Plutarque semble exclure. Nous pensons avec Georges Roux, o.c. (supra n. 1), p. 56, et Stella Georgoudi, o.c. (supra n. 1), p. 355-361, que les fonctions de prophète et de prêtre ont dû, le plus souvent du moins, être assumées par des individus distincts. Un témoignage de Plutarque à propos du prophète Nicandre (386 B-D) semble néanmoins impliquer qu'un même personnage a pu être successivement prophète et prêtre au long de sa carrière delphique.

13. Enthousiasmós, 397 C, 404 E, 430 B. Le terme implique la présence d'une puissance divine ou démonique dans le corps de celui qui est proie à l'enthousiasme.

14. de Pyth. or. $397 \mathrm{C}$ : le dieu phôs en têi psuchêi poiê̂ pròs tò méllon. 
au demeurant peut-être pas plus qu'on ne le faisait d'ordinaire dans des circonstances semblables, mais cette fois-là, les choses ont mal tourné. Le témoignage est d'autant plus exceptionnel et fascinant qu'il se réfère à un événement contemporain de Plutarque, même si lui-même n'était pas présent. On peut aisément imaginer son retentissement dans le petit monde des familles « respectables et honnêtes ${ }^{15}$ de Delphes qui donnaient au sanctuaire ses pythies et l'essentiel de son personnel, les inquiétudes et les discussions qui ont pu en résulter, comment aussi il était de nature à susciter la réflexion d'un homme à la fois pieux, philosophe et «intelligent » 16 comme Plutarque. Nous ne manquons pas d'être surpris que l'événement ait été si peu commenté dans l'abondante littérature moderne consacrée à Delphes, en dehors de la discussion technique du rôle des prophètes et des hosiö̈ ${ }^{17}$. Nous y verrions volontiers le signe de la place relativement marginale qu'y tient l'approche anthropologique des processus oraculaires ${ }^{18}$. Citons in extenso le récit de Plutarque ${ }^{19}$.

\section{UNE CONSULTATION DÉSASTREUSE}

«Quand donc la faculté imaginative et divinatoire (phantasiastikè kaì mantikè dúnamis) se trouve bien ajustée à l'état du souffle (pneúmatos krâsin), comme à un remède adéquat (pharmákou), alors les prophètes éprouvent nécessairement l'enthousiasme (tòn enthousiasmón) ; mais, quand il n'en est pas ainsi, l'enthousiasme ne se produit pas, ou bien il se produit de manière déréglée (paráphoron ${ }^{20}$, non sans atteinte et sans

15. $405 \mathrm{C}$, trad. Robert Flacelière (nomínos kaì kalôs).

16. Paul Veyne dixit.

17. Ernest Will, «Sur la nature du Pneuma delphique», Bulletin de correspondance hellénique, 66-67, 1942-43, p. 169, en faisait une «pieuse historiette », une thèse critiquée à juste titre par Robert Flacelière. Ajoutons que quoiqu'il se soit passé dans l'adyton, c'est la manière dont le prêtre Plutarque et le personnel du sanctuaire lisent l'événement qui est décisive.

18. Voir toutefois, Giulia Sissa, Le corps virginal, Paris, Vrin, 1987, p. 27-75 ou Lisa Maurizio, "Anthropology and Spirit Possession: A reconsideration of the Pythia's role at Delphi", Journal of Hellenic Studies, 115, 1995, p. 69-86.

19. def. 438 A-B. Nous donnons la traduction de Robert Flacelière que nous avons modifiée pour quelques passages décisifs.

20. Paráphoros, "qui s'écarte, vacille, vagabonde », chez Hippocrate, Prorrhétiques 70, « qui égare ou dérange l'esprit ». 
trouble (ouk akéraion ${ }^{21}$ kaì taraktikón), comme nous savons que cela est arrivé pour la pythie qui est morte tout récemment (hósper ísmen epì tês énagchos apothanoúses Pythías). Des consultants étant venus d'un pays étranger, la victime, à ce qu'on raconte, avait reçu les premières aspersions sans bouger et sans s'émouvoir (akíneton kaì apathés), puis comme les prêtres redoublaient de zèle et la pressait à l'envi, à force d'être inondée et comme noyée, elle finit à grand-peine par se rendre. Or qu'arriva-t-il à la pythie ? Elle ne descendit dans le lieu prophétique (eis tòn mantềon), dit-on, qu'avec répugnance et aversion (ákousa kaì apróthumos). Dès ses premières réponses, il fut manifeste, à sa voix rauque (têi trachúteti tês phonês), qu'elle ne se remettait pas de son trouble et qu'elle ressemblait à un vaisseau désemparé, étant pleine d'un souffle muet et mauvais (alálou kaì kakoû pneúmatos oûsa pléres). À la fin, complètement bouleversée (ektarachtheîsa), elle s'élança vers la sortie avec un cri insensé et effrayant (metà kraugês asémou kaì phoberâs), et se jeta à terre, mettant en fuite non seulement les consultants, mais encore le prophète Nicandre et ceux des hosiö̈ qui se trouvaient là. Rentrés quelques instants après, ils la relevèrent alors qu'elle avait repris ses esprits (émphrona) ${ }^{22}$ et elle ne survécut que peu de jours. »

À l'intérieur du dialogue de Plutarque, l'anecdote est intégrée à une discussion philosophique concernant les pouvoirs du pneuma et la faculté imaginative et mantique. C'est cette dimension théorique qui est généralement prise en compte par les commentateurs, avec ses corollaires : la critique des épicuriens et des stoïciens, la défense

21. Akéraion, « intact, non entamé, non souillé ». Le terme est peut-être plus signifiant qu'il ne semble. Par rapport à la théorie du pneuma, il renvoie à la physique des mauvais mélanges, mais du point de vue religieux, il pourrait suggérer une impureté (cf. Euripide, Hélène 48; Oreste 922; Aristote, Histoire des animaux 605 a 15). Dans une nouvelle traduction française des Dialogues Pythiques, très richement annotée, qui est parue alors que nous achevions cet article, Frédérique Ildefonse lit le passage d'une manière qui nous semble très convaincante : l'enthousiasme se produit « de manière sauvage, impure et perturbante $»$.

22. L'établissement du texte pose ici problème ; dans son édition de la Collection des Universités de France, Robert Flacelière a retenu la leçon ékphrona (Turnèbe), " hors d'elle-même », qu'il traduit "sans connaissance », mais la plupart des manuscrits portent émphrona, «qui a toute sa raison ». Ouk émphrona peut désigner par contraste un état de possession violente. $\mathrm{Si}$ l'on admet cette leçon, comme le fait Frédérique Ildefonse, on pourrait donc comprendre que la pythie a retrouvé ses esprits après la crise. Toutefois, si Plutarque juge utile de préciser que la pythie a toute sa tête au moment où les prophètes et les Hosioï prennent soin d'elle, c'est probablement que la chose n'allait pas de soi dans un tel contexte. 
de la providence, les relations entre causalité naturelle et divine... Plusieurs remarques s'imposent néanmoins. Le de defectu oraculorum est un dialogue aporétique où les théories expliquant le processus mantique ou le déclin de certains oracles sont données comme des hypothèses de recherche dont les interlocuteurs examinent les éventuelles conséquences théologiques. Les objections que suscite la conceptualisation trop étroitement aristotélicienne, «naturaliste », du discours de Lamprias sont à cet égard emblématiques : elle risque de réduire à néant le rôle des puissances divines et démoniques dans le processus divinatoire ${ }^{23}$. L'intéressé admet aussitôt les conséquences potentiellement impies de son discours et, prenant à son compte la critique de sa théorie encore inaboutie ou incomplète, il reformule le problème en proposant une hypothèse qui élimera la difficulté précédente ${ }^{24}$. Les protagonistes sont engagés dans une discussion qui avance d'apories en apories pour se conclure sur une invitation à approfondir la recherche : « Voilà des problèmes que nous devons fréquemment, vous et moi, examiner à nouveau, parce qu'ils suscitent beaucoup d'objections et d'hypothèses contradictoires » ${ }^{25}$. Au cœur de cette maïeutique platonicienne, la question de la pratique divinatoire, institution fondée sur la tradition (tà pátria) et comme telle incontestable, reste centrale. La place de l'anecdote dans le dialogue est en elle-même signifiante : elle vient juste à la fin, presque en conclusion, comme une question ouverte qui justifie l'invitation à poursuivre l'examen à nouveaux frais. Et c'est à l'aune des pratiques delphiques que s'éprouve la validité des hypothèses : « quand nous offrons le sacrifice préliminaire (...) nous ne faisons rien de contraire aux idées que je soutiens (oudé... enantá tôi lógoi toútoi práttomen) $\gg^{26}$.

Les préoccupations du prêtre font quelquefois explicitement surface dans le tissage rhétorique du dialogue. Le récit de la mort de la Pythie est immédiatement suivi de considérations pratiques

23. de defectu $434 \mathrm{~F}-435 \mathrm{E}$.

24. de defectu 435 E - 438 D. Voir Daniel Babut, Parerga o.c. (supra n. 3), p. 483.

25. de defectu $438 \mathrm{D}$.

26. de defectu $437 \mathrm{~A}$. 
concernant la pureté (hagneía) des pythies en général : "C'est pour cela qu'on garde la Pythie pure de toute union charnelle et complètement isolée de tout contact et de toute relation avec des étrangers. Voilà pourquoi aussi, avant la consultation, on recueille les signes (tà semeîa) de la volonté divine, dans la pensée que le dieu sait bien à quel moment la prophétesse, se trouvant dans l'état et la disposition convenables, supportera sans dommage l'enthousiasme (ablabôs hupomeneî tòn enthousiasmón). » ${ }^{27}$ Le propos constitue une exégèse raisonnée des prescriptions rituelles auxquelles le personnel oraculaire se conforme par tradition. Le lien logique entre la question de la chasteté des pythies et la mort de celle qui n'a pas tenu compte du signe préalable n'apparaît que si l'on prend en compte les problèmes auxquels est confronté le prêtre en charge d'une consultation mantique fondée sur l'enthousiasme, sur la «possession » de la prophétesse par l'instance dont elle est le porte-parole. Toute la discussion sur le pneuma présuppose l'expérience de la variabilité des dispositions de la Pythie, de sa disponibilité et de sa vulnérabilité en présence de la puissance mantique qui s'exerce en elle. Même si l'enthousiasme n'est pas folie, si l'image d'une Pythie « déchaînée » et « hors d'elle » est, sauf cas de dysfonctionnement majeur, incompatible avec le témoignage de Plutarque, le prêtre de Delphes ne cesse de souligner combien la prophétesse est affectée par le processus oraculaire. « Dès qu'elle s'éloigne du trépied et du souffle mantique, elle retrouve son calme et sa tranquillité (en galénei kaì hesuchíai) » ${ }^{28}$. «Incapable de rester passive (hesuchían) et de s'offrir immobile (akineton) et tranquille à celui qui la meut, elle répand le trouble en elle-même, comme sur une mer démontée, par l'effet des mouvements et des affections (kinémasi kaì páthesi) qui l'agitent dans son fond $»^{29}$. L'exigence raisonnable et naturelle d'une absence de trouble et de passions et celle, rituelle, d'une chasteté continue pendant toute la durée de son sacerdoce, explicitent, de manière complémentaire, la

27. de defectu $438 \mathrm{C}$.

28. Dialogue sur l'amour 759 B. Voir aussi 763 A.

29. de Pyth. or. $404 \mathrm{E}$, trad. Robert Flacelière, modifiée. Nous discutons le passage, d'interprétation très délicate dans notre article « Ménades, Pythies et autres possédés », Asdiwal 2, 2007, à paraître. 
situation dans laquelle l'accomplissement même du rite place la Pythie. C'est dire que les questions soulevées par la mort d'une pythie, par l'échec ou le dérèglement toujours possibles de la relation entre le dieu et sa prophétesse mettent d'abord en cause les savoirs du praticien du rituel ; elles contribuent néanmoins à féconder et orienter les spéculations du philosophe.

\section{PiéTÉ ET SPÉCUlation PHILOSOPHIQUe}

Une première conclusion s'impose. Du point de vue plutarquéen, l'analyse critique et l'effort d'explication « rationnelle » des processus mantiques se fondent à la fois sur les exigences de l'enquête philosophique et sur celles de la pratique rituelle. Ainsi pouvons-nous saisir au mieux l'unité d'une figure que les approches des différentes disciplines intéressées par son œuvre, histoire des religions, histoire de la philosophie, histoire des littératures, tendent à difracter en images incompatibles. Dès que sont en jeu les rapports entre les hommes et les dieux, les préoccupations du prêtre respectueux des traditions ancestrales et celle du philosophe platonisant se rejoignent. Les scrupules religieux de Plutarque, son eusébeia et son hosiótes, créent une dynamique spéculative dont témoigne la discussion entre Sérapion et Philinos dans le dialogue Sur les oracles de la Pythie ${ }^{30}$.

«Il ne faut pas combattre contre la divinité (máchesthai pròs tòn theón), ni détruire en même temps que la mantique la providence et le divin (anaireîn metà tês mantikês háma tèn prónoian kaì tò theîon), mais il faut rechercher les solutions des contradictions apparentes (tôn hupenantiousthai dokoúnton lúseis epizeteîn) et ne pas abandonner la pieuse attitude de nos ancêtres (tèn dè eusebê kaì pátrion mè proíesthai pístin ${ }^{31}$ ). »

30. $402 \mathrm{E}$.

31. Une traduction conventionnelle de pístis par «croyance» ou «foi » ne rend pas raison du respect des pratiques rituelles qu'implique la notion, c'est dans l'attachement scrupuleux aux rites que se joue la «foi » et le lien qui unissent une communauté humaine et ses dieux. Si la pístis consiste aussi dans la reconnaissance et dans le respect des représentations traditionnelles sur les dieux (Dialogue sur l'amour 756 B), c'est que ces dernières soutiennent la piété. En cela elles sont inséparables de la pratique. C'est dans les sanctuaires, lors de l'accomplissement des sacrifices et des rites que s'éprouve au plus point 
À ces préoccupations qui sont placées dans la bouche du stoïcien Sérapion, le pythagoricien Philonos répond :

«Tu as raison. Nous ne désespérons pas de la philosophie, comme si elle était entièrement perdue et anéantie ».

Dans son effort pour résoudre les contradictions apparentes, le philosophe préserve la piété ancestrale, il défend l'hosiótes traditionnelle (une notion qui présuppose, dans ses occurrences plutarquéennes, un accomplissement scrupuleux des rites ${ }^{32}$ ) contre les deux attitudes contradictoires qui la menacent, la superstition (deisidaimonía) et l'athéisme (atheótes) ${ }^{33}$. À la première, Plutarque avait consacré un petit opuscule alors qu'il n'était encore qu'un jeune sophiste prometteur ${ }^{34}$; elle est une des causes des malheurs de Nicias qui s'est avéré incapable de prendre les bonnes décisions lors de l'expédition athénienne en Sicile ${ }^{35}$; mais on peut s'en prémunir en se forgeant des idées justes sur les dieux et la manière dont ils interviennent dans les affaires humaines ${ }^{36}$. Quant à l'athéisme, il résulte de tout discours qui ébranle les fondements de la tradition, « met en

la présence des dieux : De l'impossibilité de vivre agréablement en suivant Épicure, $1101 \mathrm{E}$, commenté par Claudio Moreschini, «Religione e filosofia in Plutarco », Plutarco e la Religione, Italo Gallo (éd.), Naples, M. d'Auria, 1995, p. 47-48.

32. Sur Isis et Osiris, $355 \mathrm{C}:$ « accomplis en tous points les rites traditionnels (tôn hierôn nenomisména) ». Pour une première approche de la notion, voir Jean Rudhardt, Notions fondamentales de la pensée religieuse et actes constitutifs du culte dans la Grèce classique, Paris, Picard, $1992^{2}$, p. 30 et ss. Voir aussi les remarques critiques sur le sens de tà hósia chez Plutarque dans André-Jean Festugière, Études de religion grecque et hellénistique, Paris, Vrin, 1972, p. $170-173$.

33. Sur Isis et Osiris, 355 C-D ; 378 A : « certains se fourvoient complètement et glissent dans la superstition; d'autres fuyant les fondrières de la superstition, vont à l'opposé se jeter sans y prendre garde, dans le gouffre de l'athéisme », trad. Christian Froidefond, Paris, Les Belles Lettres, 1988, (Collection des Universités de France).

34. Voir Louise Bruit, Le commerce des dieux, Paris, La Découverte, 2001, p. 170-171; 133-144 (pour les portraits contrastés du superstitieux Nicias et de l'impie Alcibiade dans les Vies), et surtout P. Veyne, o.c. (supra n. 5), p. 676-681.

35. Vie de Nicias 23.

36. Sur Isis et Osiris, 355 C-D. 
mouvement ce qui ne doit pas être mû » (tà akíneta kineîn) ${ }^{37}$ selon une formule qui définit, à travers toute l'histoire du platonisme, de Platon aux derniers néoplatoniciens, les atteintes aux patría (aux traditions établies) qui fondent les relations entre les hommes et les $\operatorname{dieux}^{38}$.

L'examen critique des doctrines philosophiques relatives à la divination participera donc de cet acte de piété qu'est la recherche de la vraie nature des dieux. «Prions-les, et eux seuls, d'accorder à notre recherche, dans la mesure où l'homme peut y atteindre, la connaissance de leur propre nature. ${ }^{39} \mathrm{Si}$ l'épicurisme, qui explique l'exactitude des prophéties par une rencontre de hasard et une conjecture plausible, est par excellence la philosophie qui dispose à l'athéisme ${ }^{40}$, la réduction des dieux à des éléments naturels, telle qu'impliquée par la physique stoïcienne et par un certain type de lecture allégorique des mythes n'en est pas moins périlleuse ${ }^{41}$. Certaines conceptions de la nature ont pour conséquence que les processus oraculaires tels que les rites traditionnels les mettent en œuvre pourraient ne plus être regardés comme une pieuse communication entre hommes et dieux. Même si elle la tient pour efficace, une appréhension purement naturaliste de la mantique comporte une part d'impiété. La confusion qui s'empare de Lamprias - le frère de Plutarque, en charge, lui aussi, d'un sanctuaire oraculaire, le Trophonion de Lébadée - lorsque, dans le de defectu, Ammonios lui fait prendre conscience des implications de son discours, en est le signe le plus visible. «Pour moi, tes objections ne m'ont pas seulement ébranlé, elles m'ont bouleversé, à l'idée que dans une assemblée si nombreuse et si prestigieuse, je puisse sembler en

37. Dialogue sur l'amour $756 \mathrm{~B}$.

38. Platon, Théétète 181 A 8-B 1; Porphyre, De l'abstinence, 1. 4. 1 ; Marinos, Vie de Proclos, 30, où l'expression vise l'impiété des Chrétiens, destructeurs des cultes traditionnels.

39. Sur Isis et Osiris, $351 \mathrm{C}$, trad. Christian Froidefond ; voir aussi 355 C.

40. de defectu $434 \mathrm{D}-\mathrm{E}$; De l'impossibilité de vivre agréablement en suivant Épicure, $1086 \mathrm{C}$ et ss.

41. Sur Isis et Osiris, $377 \mathrm{D}$ : « il faut se garder, et c'est là l'essentiel, avec la plus extrême vigilance, de réduire sans le vouloir le divin, de le circonscrire dans les vents, les cours d'eau, les semailles (...) et, par là-même, de l'abolir, tels ceux qui appellent le vin Dionysos et le feu Héphaïstos, comme Cléanthe... ». 
dépit de mon âge, me parer du caractère persuasif du raisonnement pour détruire et ébranler les coutumes vraies et saintes à propos du divin (anaireîn ti kaì kineîn tôn alethôs kaì hosíos perì toû theíou nenomisménon). $»^{42}$ La question du rapport entre piété et philosophie ne se joue donc pas chez Plutarque, ainsi qu'on l'a si souvent écrit, entre foi ou croyances religieuses et raison ${ }^{43}$, mais entre enjeux de la pratique rituelle et spéculation théorique.

L'investigation philosophique est, pour une part du moins, motivée par un souci d'eusébeia et d'hosiótes. « Nous devons prendre pour guide (mustagogón) le raisonnement philosophique, examiner et comprendre avec piété (hosíos dianoeîsthai) chaque récit et chaque acte rituel (tôn legoménon kaì droménon hékaston), de sorte que (...) nous évitions de nous fourvoyer et d'interpréter à contresens les excellentes prescriptions du cérémonial des sacrifices et des fêtes (hà kalôs hoi nómoi perì tàs thusías kaì tàs heortàs étaxan hetéros hupolambánontes examártomen). ${ }^{44} \mathrm{Il} \mathrm{s'agit} \mathrm{là} \mathrm{de} \mathrm{pénétrer} \mathrm{la} \mathrm{pensée}$ et l'intelligence immanentes au rite, « de dégager la vérité dont les cultes sont porteurs (perì tês en autô̂s aletheías) ${ }^{45}$. Très concrètement aussi, il s'agit de ne pas se méprendre dans l'exécution du

42. de defectu $435 \mathrm{E}$, trad. Frédérique Ildefonse. La traduction de Robert Flacelière de nenomisménon par croyances laisse échapper l'enjeu même des objections d'Ammonios, 435 A-B : la physique exposée par Lamprias détourne notre pensée des dieux et nous met dans l'esprit une conception des causes semblable à celle du cyclope d'Euripide qui se refusait à sacrifier aux dieux (oú thúein tô̂s theô̂s) et ne sacrifiait qu'à lui-même et à son ventre.

43. Voir par ex. Robert Flacelière, "Plutarque et la Pythie », Revue des études grecques, 56, 1943, p. 72-111, ou l'étude si fine et précieuse de Daniel Babut, « Du scepticisme au dépassement de la raison : philosophie et foi religieuse chez Plutarque », Parerga o.c. (supra n. 3), p. 549-581. Héritage du débat foi raison, tel que revivifié par la renaissance thomiste de la première moitié du $\mathrm{XX}^{\mathrm{e}}$ siècle ? La conclusion de l'article de Robert Flacelière le laisserait penser : "Avec l'âge et sous l'influence de la cité sainte qui était devenue sa seconde patrie et du sanctuaire dont l'atmosphère devait imprégner de plus en plus une âme aussi religieuse que la sienne, il en vint à se sentir plus "théologien" encore que "philosophe", et sans renier la philosophie, à ne plus voir en elle que l'humble servante de la théologie, ancilla theologiae». Sur ce que peut vouloir dire « être croyant » au temps de Plutarque, voir les belles pages de Paul Veyne, o.c. (supra n. 5), p. 672 et ss.

44. Sur Isis et Osiris, 378 B, trad. Christian Froidefond, modifiée.

45. Sur Isis et Osiris, $378 \mathrm{C}$. 
rituel, qui, pour être quelquefois routinière, n'est pas pour autant «mécanique ${ }^{46}$. Elle suppose des savoirs pour lesquels, dans un processus aussi complexe et sensible que la consultation de la Pythie, aussi lourd de conséquences que l'interprétation des signes mantiques, un homme éduqué et scrupuleux comme Plutarque mobilisera spontanément tous les instruments intellectuels que la culture de son temps met à sa disposition. En retour l'examen critique qu'impose la piété, la recherche de solutions aux contradictions qui semblent se faire jour dans la manifestation du divin, notamment dans les procédures rituelles qui l'actualisent, sont un puissant facteur d'innovation et de transformation, de réinterprétation ou d'infléchissement de la tradition.

\section{UN TRADITIONALISME NOVATEUR}

Le de defectu et le de Pythiae oraculis interrogent la manière dont les dieux agissent dans le monde à travers les médiations oraculaires. Évolution de la pensée de Plutarque ou différence de point de vue, les deux dialogues explorent les hypothèses qu'ils développent sur un mode sensiblement différent. Les incertitudes et l'absence de conclusions dans le de defectu ne s'expliquent peut-être pas tant par les perplexités d'un Plutarque encore relativement jeune que par le choix de la forme aporétique du dialogue platonicien comme du moule rhétorique le plus apte à faire ressortir les insuffisances des hypothèses discutées. L'idée d'une providence divine « répondant » à la dépopulation de la Grèce, par une restriction des canaux mantiques par lesquels elle choisit de se manifester, les considérations sur la mortalité des daímones nécessaires au processus oraculaire ou sur la raréfaction du pneuma, ne sont donnés que comme des explications partielles et conjecturales du déclin des oracles. Elles explorent la contradiction entre ce qui pourrait apparaitre comme un mauvais fonctionnement de la communication entre hommes et dieux et la

46. Voir, à titre de comparaison, côté romain, l'analyse que John Scheid, Quand faire, c'est croire, Paris, Aubier, 2005, p. 64 et ss, propose de la manière dont les frères Arvales ne cessent de reformuler les listes de divinités dans les protocoles de leurs sacrifices. 
prónoia, le souci dont les immortels font montre envers le monde. Le tardif de Pythiae oraculis ${ }^{47}$ propose une solution qui allie la longue expérience du prêtre de Delphes à une conception platonicienne de la conjonction d'une cause divine et d'une cause naturelle ${ }^{48}$. L'impulsion du mouvement est le fait d'Apollon qui use de la Pythie comme d'un instrument (órganon) docile ${ }^{49}$ qui ne doit opposer aucune passion propre à l'action par laquelle «le dieu produit dans son âme les "représentations" (phantasías) et la lumière qui éclairent l'avenir ${ }^{50}$. Le dieu «signifie » (semaínei) et révèle les conceptions divines (noéseis) que la pythie réfléchit comme la lune la lumière du soleil ${ }^{51}$, en en formulant un pâle reflet avec ses mots mortels. L'énonciation pythique est à la fois image, fidèle mais affadie de la pensée d'Apollon et signe. « Le maître à qui appartient l'oracle ne dit ni ne cache ; il indique », selon une sentence d'Héraclite que Plutarque cite et réinterprète au moment le plus décisif de son argumentation ${ }^{52}$. Au plus près du processus rituel, l'effort d'élucidation critique de l'enthousiasme apollinien apparaît comme une des sources majeures de la rélaboration théorique des relations entre signe, langage et image que Plutarque développe selon une conceptualisation et une problématique typiques du Moyen Platonisme, notamment dans son dialogue sur le démon de Socrate, où la question est traitée dans le cadre d'une théorie plus générale de la communication avec les dieux et de l'homme divin ou démonique (theîos) ${ }^{53}$. Quant aux signes que le divin prodigue à la multitude, ils constituent la matière même de la divination (tò theîon semeîa dídosin, ex hôn he legoméne mantikè sunésteke $)^{54}$.

47. Nous nous rallions volontiers à une datation autour de 125 .

48. Il faut toutefois noter qu'au-delà d'importantes différences dans le traitement des fonctions du souffle prophétique entre les deux dialogues, la théorie platonicienne de la double causalité apparaît dans la dernière réponse de Lamprias dans le de defectu, $436 \mathrm{D}-\mathrm{F}$, ce qui interdit de surdéterminer l'hypothèse d'une évolution en profondeur de la pensée de Plutarque.

49. 404 C-D.

50. $397 \mathrm{~B}-\mathrm{C}$.

51. $404 \mathrm{C}$.

52. 404 D. Voir Giulia Sissa, o.c.(supra n. 18), «Pythie lunaire », p. 51-58.

53. Voir 588 C-589 F ; 592 C-F ; 593 A-D.

54. $593 \mathrm{D}$. 
Il faut donc admettre qu'une part importante des spéculations de Plutarque, et souvent en ce qu'elles ont de plus original, s'enracine dans son analyse des processus divinatoires et des modalités de la communication entre dieux et hommes, que sa « religion » est partie intégrante de l'élaboration d'un discours philosophique et rationnel. Ces termes, sans doute inévitables, sont toutefois porteurs d'ambiguïté : la religion de Plutarque ne signifie pas tant les croyances et l'attitude personnelles de l'homme et du philosophe que les devoirs du prêtre et la pratique des rites, tels toutefois qu'ils portent l'exigence d'une théologie ${ }^{55}$ et d'une herméneutique. Le discours rationnel, sous sa forme philosophique et spéculative, est commentaire et exégèse sur ce qui se passe dans le rite, sur les divers phénomènes mantiques : inspiration, prodiges ${ }^{56}$, signes ou rêves livrés à l'interprétation ${ }^{57}$, et sur les puissances divines et démoniques qui y sont impliquées. Ainsi, à propos du rôle des Muses dans le processus divinatoire, le polythéiste Plutarque cherche-t-il à clarifier les relations panthéoniques induites par le rituel et par la topographie du sanctuaire delphique ${ }^{58}$ : la question du rapport entre eau et Muses est d'abord affaire de rite : «Il y avait là un sanctuaire des Muses autour de la résurgence de l'écoulement ; c'est la raison pour laquelle on utilisait cette eau-là pour les libations et [les lustrations], comme le dit Simonide (...). Simonide encore, dans un autre passage, s'adressant à Clio, l'appelle d'une façon un peu affectée, "la pure gardienne des eaux lustrales", "vers qui montent mille prières (...)". Eudoxe avait donc tort de se fier à ceux qui déclarent qu' on appelait cette eau-là eau du Styx. » Aussitôt après, Plutarque explicite les fonctions des Muses : « Si on les installa ici comme assistantes et

55. de defectu $410 \mathrm{~B}$, où Cléombrote est présenté comme tenant l'enquête historique pour le matériau (húlen) de la philosophie dont la théologie est la fin et l'accomplissement (télos).

56. Voir notamment de Pyht. or. 397 E-398 C, (prodiges liés aux offrandes déposés dans les sanctuaires), Sur le démon de Socrate 594 E (éclair sans tonnerre annonçant la libération de Thèbes en 379), ou Vie d'Alexandre, 14, 8 (débat sur une statue d'Orphée couverte de sueur)...

57. Fredrerik E. Brenk, "The Dreams of Plutarch's Lives", Latomus, 34, 1975, p. 336-349.

58. de Pyth. or. 402 C-D, trad. Georges Roux, o.c. (supra n. 1), p. 140-141, modifiée. 
comme gardiennes de la divination (parédrous tês mantikês kaì phúlakas), à côté de la fontaine et du sanctuaire de Terre, à laquelle appartenait, dit-on, l'oracle, c'est parce que l'avenir y était chanté sous une forme poétique et rythmée ». Le texte perd beaucoup de sa densité signifiante si on ne le lit pas dans la perspective d'un «panthéon» mis en place au cour de la pratique et de l'espace cultuels ${ }^{59}$. Les noms que Plutarque donne par ailleurs aux Muses delphiques, qui sont ceux-là mêmes des cordes de la lyre, Nété (Haute), Mésé (Moyenne), Hypaté (Basse), témoignent aussi de ce que leurs prérogatives étaient traditionnellement à Delphes l'objet de spéculations complexes ${ }^{60}$.

Ne serait-ce que par l'ampleur et la richesse des constructions qu'elle met en jeu, l'exégèse « rationnelle » des rites conduit le pieux philosophe à adopter vis-à-vis de son objet une attitude plus distante, plus réflexive, et à produire une herméneutique originale et novatrice de « la vérité qu'ils contiennent » ${ }^{61}$. Le traité Sur Isis et Osiris est à cet égard emblématique : son extraordinaire sophistication mythique «en clef égyptienne » ne contredit en rien la fonction qu'il se donne explicitement : il constitue un commentaire exégétique particulièrement ample de la pratique rituelle ${ }^{62}$, susceptible, par ses détours mêmes, d'éclairer la dédicataire, son amie Cléa qui, à la tête du collège des Thyades, est en charge d'un ensemble de rites dionysiaques ${ }^{63}$. «Qu'Osiris ne fasse qu'un avec Dionysos, qui pourrait le savoir mieux que toi, la supérieure des Thyades de Delphes, qui fus consacrée par tes pères et mères aux rites osiriens ? ${ }^{64}$ Non

59. Voir Philippe Borgeaud, « Manières grecques de nommer les dieux », Colloquium Helveticum, Cahiers suisses de littérature comparée 23, Berne, 1996, p. 21 : «La pratique religieuse exige ainsi chaque fois, et en chaque lieu, une redéfinition non seulement de l'entité particulière à laquelle s'adresse le rite, mais de l'ensemble du panthéon dont cette entité se trouve solidaire ».

60. Propos de table 745 B.

61. Sur Isis et Osiris 378 C.

62. Voir notamment Sur Isis et Osiris 378 A-F.

63. Voir Marie-Christine Villanueva Puig, « À propos des Thyades de Delphes », L'association dionysiaque dans les sociétés anciennes, Table ronde, Rome, 24-25 mai 1984, Olivier de Cazanove (éd.), Rome, 1986, (Collection de l'École Française de Rome, 89), p. 31-51.

64. $364 \mathrm{E}$. 
seulement des attributs et des qualités de Dionysos, mais aussi certaines de ses fêtes locales, Nyctélies et éveil du dieu dans son liknon par les Thyades et les Hosiö réunis, sont explicités en référence à des pratiques et à des mythes attribués aux Égyptiens ${ }^{65}$. Un réseau de significations secondes est ainsi constitué, en prise sur le rite, éventuellement susceptible d'en inspirer la réinterprétation ou la réorganisation.

C'est donc aussi à la production de nouvelles variantes mythiques que contribuent travail critique, exégèses et spéculations. La version du mythe de la mort de la Sibylle que Plutarque place dans la bouche de Sérapion doit être mise en rapport avec sa réflexion sur les médiations, notamment démoniques, qu'impliquent les processus mantiques ${ }^{66}$. « Il rappela alors les vers dans lesquels elle a chanté son propre sort, en proclamant que même morte, elle ne cesserait pas de prophétiser, mais qu'elle passerait dans la lune et en suivrait les évolutions, en s'identifiant au prétendu visage que l'on y observe, son souffle mêlé à l'air, et sans cesse errant dans le monde, produirait les voix et les présages (phémais kaì kledósin), son corps enfin, décomposé dans la terre, ferait pousser l'herbe et les plantes, nourriture des animaux sacrés (hierà thrémmata), dont les viscères (splágchnon), avec leurs couleurs, leurs formes et leurs qualités diverses, manifesteraient l'avenir aux hommes. » Entre production mythique et philosophie, les spéculations sur la sibylle - elles-mêmes fondées sur une vénérable prophétie - renvoient à la pratique cultuelle. Contre l'épicurien Boethos, elles suggèrent, dans le contexte narratif du dialogue, une conception de la rencontre entre signes et événements qui ne repose pas sur le hasard mais sur une « économie » prophétique à l'œuvre à travers l'univers. Elles constituent un mythe qui rend compte à la fois des vertus mantiques inhérentes à la nature dans leur relation à une figure inspirée et, à travers elle, à la providence divine ${ }^{67}$, et des rites fondés tant sur les

65. 364 F-365 A.

66. de Pyth. or. 398 C-D, trad. Robert Flacelière, modifiée.

67. Sur les conceptions plutarquéennes relatives aux interventions divines dans les affaires humaines, voir Daniel Babut, Plutarque et le stoïcisme, Paris, PUF, 1969, p. 473-483. 
bruits et les voix ${ }^{68}$ que sur l'observation des viscères ${ }^{69}$. Elles ne proposent rien moins qu'une articulation en profondeur entre ces aspects complémentaires et centraux de la pratique rituelle que sont l'élevage de troupeaux sacrés, le sacrifice et la mantique - paroles et signes.

La piété du prêtre et celle du philosophe, qu'il argumente ou reformule les mythes, sont solidaires. Pour les Happy Few qui y accèdent, « la quête de la vérité, en premier lieu celle qui concerne les dieux, est une aspiration au divin (theiótetos órexis), telle que l'accès aux choses sacrées (análepsin híeron) tient au savoir et à la recherche (máthesin kaì zétesin), qui sont une pratique comportant une hosiótes supérieure (érgon hosióteron) à toute lustration et à tout service dans un sanctuaire (hagneías páses kaì neokorías) $\gg^{70}$. Une fois encore, il convient de ne pas projeter sur une telle affirmation l'idée aisément anachronique d'une supériorité de la philosophie et de la connaissance rationnelle comme telles sur la «religion», notamment comme pratique des rites. Ce serait négliger le contexte énonciatif dans lequel la formule fait sens, comme portique à l'immense effort d'exégèse des rites et des mythes qu'est le traité sur Isis et Osiris, négliger aussi la portée des termes choisis par Plutarque. En traduisant análepsin híeron par « révélation du divin » ou par $«$ consideration of sacred subjects ${ }^{71}$, on court le risque d'oublier que les hierá dont il est question se rapportent aussi bien aux dieux dans leur nature que dans leurs rites. Simplement les rites sont ici considérés du point de vue de la vérité qu'ils recèlent ${ }^{72}$,

68. Sur la manière dont la rencontre avec la voix prophétique peut être organisée par le rite, voir Dominique Jaillard, « Hermès et la mantique grecque », Signes, rites et destin, Actes de la table ronde de Paris, 16-17-18 octobre 2005, Stella Georgoudi, Renée Koch Piettre, Francis Schmidt (éds.), à paraître, et sur la voix de la Sibylle, Sabina Crippa, « Figures du sibullaínein », La sibylle. Parole et représentation, Monique Bouquet et Françoise Morzadec (éds.), Presses universitaires de Rennes, Rennes, 2004, p. 102-103.

69. Sur la valeur et la place centrale des viscères (splánkna) parmi les hierá, voir Jean Rudhardt, o.c. (supra n. 32), p. 255-256, 262-263, Marcel Detienne, Dionysos mis à mort, Paris, Gallimard, 1977, p. 174-179.

70. Sur Isis et Osiris $351 \mathrm{E}$.

71. Christian Froidefond, Collection des Universités de France ; Frank Cole Babbitt, Loeb Classical Library.

72. Sur Isis et Osiris $378 \mathrm{C}$. 
qu'il est de la tache du prêtre conscient de ses devoirs d'éclairer par la pratique de la philosophie. Si la recherche de la vérité concernant les dieux est plus hósios, c'est à plus conforme à l'ordre divin, et comporte une piété plus grande pour celui qui s'y livre, c'est qu'elle traite les rites en prenant en compte la plénitude du sens qu'un examen éclairé reconnaît en eux. La philosophie est piété envers les rites et les traditions; dans la dense formule de Plutarque, elle n'est pas supérieure à la prêtrise, mais à la neocoría, qui renvoie à des fonctions davantage liées aux aspects matériels de l'entretien du sanctuaire $^{73}$. Quant à la philosophie, elle est aussi, comme mode de vie, hagneía, purification ritualisée ${ }^{74}$. Il y a bien une supériorité, celle du philosophe sur celui qui n'exerce pas sa faculté théorétique, en ce que sa discipline le rend apte à comprendre, avec la vraie nature du divin, ce que met en jeu et réalise le cérémonial des sacrifices et des fêtes ${ }^{75}$. Plutarque prolonge la vieille idée que « seuls les sages sont prêtres ${ }^{76}$, en lui donnant un élan et une dimension nouvelle à la mesure de cette conscience aiguë - qui le place au seuil d'un monde nouveau ${ }^{77}$ - que la pratique et l'intelligence de la pratique sont les deux faces solidaires d'une même piété, que la philosophie elle-même

73. Voir Stella Georgoudi, o.c. (supra n. 1), p. 360-361. La néocorie semble impliquer une relation avec le divin moins étroite que la prêtrise d'un dieu. Quelque qu'aient été les fonctions exactes du néocore à Delphes, sans doute moins modestes qu'on ne l'a quelquefois affirmé, le mot renvoie à l'idée de service et d'entretien. Le caractère honorifique de la fonction est attesté par l'épigraphie (cf. par ex. le décret de Thasos en l'honneur de la néocore Epiée, Supplememtum epigraphicum graecum 18, 1962, $\mathrm{n}^{\circ} 343$ ).

74. Voir Pierre Hadot, Exercices spirituels et philosophie antique, Paris, Albin Michel, $2002^{2}$, p. 25 et ss.

75. $378 \mathrm{C}$.

76. Diogène Laërce, Vies et doctrines des philosophes illustres, VII. 119, à propos du sage stoïcien : «Les sages sont pieux, car ils ont l'expérience des coutumes (nomínon) qui concernent les dieux. Ils sacrifieront aux dieux et sont purs (...). Les dieux s'émerveillent d'eux, car ils sont saints (hosíous) et justes envers le divin. Seuls les sages sont prêtres, car ils ont étudié les sacrifices, les fondations, les purifications et toutes les autres institutions appropriées aux dieux ».

77. Paul Veyne, o.c. (supra n. 5), p. 674 : «Plutarque trace la frontière religieuse entre "Haut Empire" et "Bas Empire" (...), entre la religiosité telle qu'elle est depuis quatre siècles et ce que sera bientôt celle d'un Aelius Aristide (qui aurait été, aux yeux de Plutarque, le Superstitieux par excellence) ». 
est culte des dieux. Avec Plutarque, nous saisissons l'émergence d'une figure neuve, celle d'un philosophe prêtre, profondément pénétré des devoirs de la fonction qu'il s'assigne, et qui jouera un rôle si important dans l'Antiquité tardive, jusque chez les derniers Platoniciens qui - les sacrifices désormais interdits, les pátria ébranlés - se voudront, tel Proclus, hiérophantes de tous les dieux ${ }^{78}$. Parce que la mantique, en ses diverses manifestations, est au cœur de la communication entre les hommes et les dieux, parce que son destin a fait de Plutarque un prêtre d'Apollon à Delphes, la divination s'est pour lui avéré un opérateur privilégié pour penser toutes les formes de relation avec les dieux, une relation que les rites institués ont le privilège de réaliser en acte.

Université de Lausanne

Institut d'Archéologie et de Sciences de l'Antiquité

Anthropole 4015

CH 1015 Lausanne Dorigny

78. Marinos, Vie de Proclos, 19. 